\title{
Toward a unified theory of aging and regeneration
}

\author{
Michael D West*,1, Hal Sternberg ${ }^{1}$, Ivan Labat ${ }^{1}$, Jeffrey Janus ${ }^{1}$, Karen B Chapman ${ }^{2}$, Nafees \\ N Malik ${ }^{1,3}$, Aubrey DNJ de Grey ${ }^{1,4}$ \& Dana Larocca ${ }^{1}$ \\ ${ }^{1}$ AgeX Therapeutics, Inc., Alameda, CA 94501, USA \\ 2Johns Hopkins University, Baltimore, MD 21218, USA \\ ${ }^{3}$ Juvenescence Ltd, London, UK \\ ${ }^{4}$ SENS Research Foundation, Mountain View, CA 94041, USA \\ *Author for correspondence: Tel.: +1 510671 8370, Ext.: 303; Fax: +1 510671 8619; mwest@agexinc.com
}

Growing evidence supports the antagonistic pleiotropy theory of mammalian aging. Accordingly, changes in gene expression following the pluripotency transition, and subsequent transitions such as the embryonic-fetal transition, while providing tumor suppressive and antiviral survival benefits also result in a loss of regenerative potential leading to age-related fibrosis and degenerative diseases. However, reprogramming somatic cells to pluripotency demonstrates the possibility of restoring telomerase and embryonic regeneration pathways and thus reversing the age-related decline in regenerative capacity. A unified model of aging and loss of regenerative potential is emerging that may ultimately be translated into new therapeutic approaches for establishing induced tissue regeneration and modulation of the embryo-onco phenotype of cancer.

First draft submitted: 13 May 2019; Accepted for publication: 5 August 2019; Published online: 28 August 2019

Keywords: acetyl-COA • aging $\bullet$ AMPK • dietary restriction $\bullet$ DNA methylation $\bullet$ epigenetics $\bullet$ mTOR $\bullet$ pluripotent stem cells $\bullet$ regeneration

Aging is often defined as a progressive deterioration of an organism over time, wherein the risk of mortality increases exponentially with age in the postreproductive years. Although everyday environmental risks from predation or infectious disease (e.g., stochastic risks) necessarily lead to increased mortality over time, they are not considered core to the definition of the aging process per se [1,2]. Thus, an important criterion of aging is that it encompasses virtually every somatic tissue type, including the gonads (though not necessarily the germ-line cells themselves, given their role in potentially perpetuating the species) [3]. In order to distinguish the aging process from damage that occurs stochastically over time, Benjamin Gompertz described aging as a process leading to an exponential increase in mortality with time, that is, $R_{m}=R_{0} e^{\text {at }}$ where ' $R_{m}$ ' represents the probability of mortality between ages ' $\mathrm{t}$ ' and ' $\mathrm{t}+1$ '. Accordingly, ' $\mathrm{R}_{0}$ ' is a constant, while 'a' represents an exponential parameter corresponding to the rate of increase of mortality with age [1,4]. Diverse species exhibit wide variations in the rate of aging as high as 50-fold, and some species even show a negligible rate of aging as determined by Gompertz equation. However, the similarities in the aging process between species and within tissues of an individual support the conclusion that what we commonly call aging is the result of a series of developmentally timed processes occurring in somatic tissues [5].

The pathogenesis of aging, like the relatively complex etiology of cancer, is commonly viewed as being rooted in multifaceted processes intrinsic to somatic cells along with additional tissue-specific influences, inherited genetic predispositions and environmental factors. However, premature aging disorders such as Hutchinson-Gilford syndrome (progeria) and Werner syndrome showing the premature appearance of various age-related changes such as graying and loss of hair, coronary heart disease, stroke and osteoporosis [6] lend support to an intrinsic genome-based pacing mechanism underlying aging. In addition, these naturally occurring aging disorders provide evidence that certain molecular pathways may alter the time course of the aging in multiple organ systems, suggesting that similar pathways may underlie aging in numerous somatic tissue types.

Future
Medicine 
Additional insights into the molecular biology of aging can be gleaned from studies of segmental (tissue-specific) genetic predispositions as well as those comparing the genotypes of individuals with varied lifespan phenotypes. The use of genome-wide gene association studies has identified loci such as those near APOE, CHRNA5, ANRIL, LPA and FTO as being correlated with a long lifespan [7]. However, identical twin studies suggest that environmentally induced epigenetic effects potentially play an important role in the onset of age-related disease as well [8]. Therefore, despite recent progress in understanding the biology of aging, the field remains largely fragmented due to the lack of a central organizing hypothesis that could provide a framework for investigating how basic upstream biological processes regulate the timing of age-related changes in tissues and the influence of these changes on the onset of age-related degenerative disease.

The goal of this review is to assemble diverse observations about the etiology of aging into a more unified mechanistic model. Thus, we suggest that numerous genetic, environmental and metabolic perturbations alter the rate of aging by shifting the balance of euchromatin/heterochromatin in regulatory regions of the genome leading to either more permissive or more restrictive patterns of regenerative gene expression. Moreover, we propose that these changes in regenerative gene expression occur in a series of developmentally timed transitions that occur globally in many cell types and in various tissues of the body and that these changes impact multiple hallmarks of both aging and cancer. Using an historical perspective, we will review these developmentally timed transitions from the perspective of the loss of germ-line immortality in somatic cells and the changes in regenerative gene expression (antagonistic pleiotropy) leading to the progressive restriction of regenerative capacity and the subsequent effects on aging. Finally, we will discuss the epigenetic nature of these transitional changes that lead to aging and how their reversal during reprogramming suggests the possibility of restoring regenerative capacity to aging tissues and organs using an approach we have termed induced tissue regeneration (iTR).

\section{Somatic restriction \& antagonistic pleiotropy}

In the 19th century, the German naturalist August Weismann described the elegant hypothesis that heredity is transmitted through an immortal lineage of cells commonly designated the 'germ line' [9]. This logically led to his proposal that since somatic cells do not carry their hereditary information forward to the next generation, they have no need for the capacity to replicate indefinitely. In other words, somatic cells are disposable from the viewpoint of evolution because there is no selective pressure for traits that would lead to their immortal replication. He therefore laid out the prediction that "death takes place because a worn-out (somatic) tissue cannot forever renew itself, and because a capacity for increase by means of cell division is not everlasting, but finite" [9].

Weismann proposed that the loss of immortal replication in somatic cells (one example of somatic restriction) associated with aging resulted from the loss of traits that were not evolutionarily advantageous (a passive process). In contrast, the evolutionary biologist George Williams proposed a model of antagonistic pleiotropy as a likely mechanism in which aging was under active selection. In his model, certain genetic traits have pleiotropic effects throughout the lifespan. Accordingly, natural selection necessarily increases the probability of those traits that lead to increased fecundity, therefore, once reproduction has occurred there is very little selective pressure to eliminate these same traits even if they exert deleterious effects on fitness or viability. As a result, the two outcomes are antagonistic, such that natural selection favored traits that led to the limited replication and regeneration of the soma despite potentially deleterious effects later in life (i.e., aging) because these same traits improved reproductive fitness early in life.

The Weismann and Williams hypotheses are not mutually exclusive. Indeed, both hypotheses have grown in acceptance since they were first published and as a result, it is now commonly accepted that mammalian aging is likely the result of a developmental program wherein somatic cells progressively lose the traits of immortal proliferation and regeneration (somatic restriction) and that such restriction is the result of active selection for increased reproductive fitness. Accordingly, selection for traits that decrease the risk of oncogenesis or increase resistance to genomic instability from endogenous retroviral elements would likely increase reproductive fitness but could also contribute to the decline of regenerative potential that is observed in later life.

The restriction of the twin traits of immortal proliferation and regeneration is designated as the 'Weismann barrier' herein as shown in Figure 1. Accordingly, Weismann's prediction that a worn-out tissue cannot forever renew itself was shown to be the case in many vertebrate species wherein scarless regenerative potential is repressed shortly after embryogenesis. In contrast, simple unicellular organisms such as Tetrahymena display replicative immortality, and a few primitive invertebrates such as hydra $[10,11]$ and planaria show no evidence of aging as determined by 


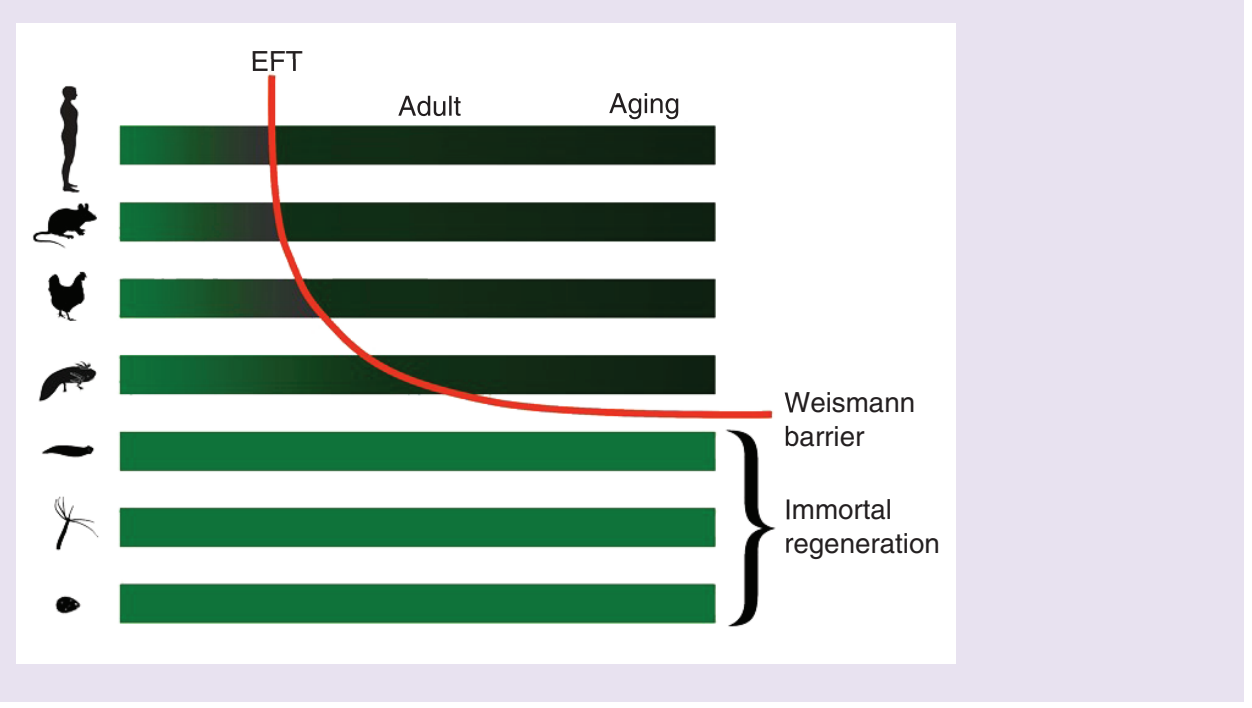

Figure 1. Evolution and the Weismann barrier. Primitive protozoa such as Tetrahymena appear to be capable of limitless reproduction in the absence of sexual recombination. Multicellular animals such as hydra and planaria likewise display profound regenerative potential with replicative immortality leading to an absence of aging as defined by Benjamin Gompertz. In vertebrates, repression of replicative immortality is commonly observed during early embryonic development and regeneration is repressed later usually around the embryonic-fetal transition defining the Weismann barrier (shown as a red line). For those examples where extensive regeneration is observed in the adult, it is common to see the adult arrested in an early developmental state as observed in axolotl pedomorphs. EFT: Embryonic-fetal transition.

an exponential rise in the risk of mortality with time. However, in general, advanced multicellularity without cancer required a phenotype that confined continual renewal to the germ line while restricting the regenerative capacity of somatic cells and tissues. As a result, as multicellular organisms evolved to even greater complexity their regenerative capacity became increasingly restricted, and as we propose herein, the restriction may occur globally in developmental stages and include numerous cell and tissue types. Thus, nearly all mammals, including humans, repress replicative immortality in somatic cells early in embryogenesis followed by a subsequent loss of regenerative potential after embryonic development is essentially complete. This loss of both immortality and regenerative potential defines the Weismann barrier (shown as red line in Figure 1).

The somatic restriction model predicts that organisms that retain juvenile properties throughout life would also retain enhanced regenerative capacity because they would never completely traverse the Weismann barrier. Accordingly, support for the model that the profound regenerative potential of primitive metazoans is retained from early ontogeny comes from studies in amphibians such as the urodeles. For example, axolotls live their adult lives in an arrested larval stage (pedomorphosis) due to the lack of appropriate thyroid hormone signaling $[12,13]$. These unusual animals show a profound regenerative potential in diverse tissues such as forebrain, jaw and heart [14-16]. In addition, they are even capable of regenerating amputated limbs through the formation of a limb bud-like blastema, a phenomenon designated as epimorphic regeneration [17]. Moreover, the observation that other anuran larvae (e.g., Xenopus) exhibit significant regenerative potential that is subsequently lost with metamorphosis supports the view that the retention of embryonic traits causes epimorphic regeneration in urodeles [18]. Furthermore, there are additional models provided by various species that retain regenerative potential into adulthood such as hemimetabolous insects that show an extended nymph-like state [19]. However, it is important to note that repeated injury to axolotls eventually leads to defective regeneration suggesting that while they may retain the profound regenerative potential of the larval state and appear to express telomerase, they may not have sufficient telomerase activity to regenerate indefinitely [20,21]. Therefore, urodeles, unlike certain more primitive organisms like planaria and hydra, do not completely escape the Weismann barrier (Figure 1). The lack of telomerase as well as other insults to the genome ultimately leads to cellular senescence, which profoundly affects regenerative capacity and tissue homeostasis later in life. 


\section{Somatic cell senescence}

Weismann's prediction that somatic cells only possess a finite capacity for cell division was validated in the 1960s through the work of Leonard Hayflick who finally provided evidence for the mortality of cultured normal diploid human fibroblasts [22]. In the following years, virtually all normal human cell types capable of cell division in vitro were shown to have only a finite capacity for cell division. Importantly, this finite replicative lifespan was determined to be a result of the number cell doublings that a cell had undergone rather than simply the passage of metabolic time [23]. However, the precise nature of this replicative clock remained to be determined for several decades.

In 1973, Alexey Olovnikov proposed that the progressive shortening of terminal DNA sequences (telomeres) was the clocking mechanism for the Hayflick phenomenon, and that immortal cells such as germ line and cancer cells utilize a terminal transferase (now designated telomerase) that reverse transcribes the tandem telomeric repeat sequences utilizing an endogenous RNA template, thereby extending the telomere length [24]. However, Olovnikov's hypothesis could not be tested until the molecular cloning, in the 1990s, of the gene encoding the catalytic component of telomerase, known as telomerase reverse transcriptase (TERT). As predicted by the hypothesis, the exogenous expression of TERT extended the lifespan of normal cells that were grown in vitro [25]. Subsequent studies demonstrated the ability of the enzyme to immortalize various somatic cell types without inducing oncogenic transformation [26]. Additional studies using a sensitive PCR-based assay for telomerase activity, known as the telomere repeat amplification protocol (TRAP) assay, demonstrated that telomerase activity was present in approximately $98 \%$ of immortal cancer cell lines (but not in any normal cultured somatic cell types), approximately $90 \%$ of malignant tumors and $100 \%$ of normal gonads tested, but not in other normal somatic counterparts [27]. As shown in Figure 2A, the pattern of TERT expression fits the model of antagonistic pleiotropy. Embryonic stem (ES) cells have not repressed TERT and, therefore, they commonly maintain telomere length near that of germ-line cells such as sperm [28,29]. However, human ES (hES) cells that are differentiated to early embryoid bodies or partially differentiated to clonal embryonic progenitor cell lines [30] have passed the pluripotency transition (PT) and therefore do not express TERT. Additional support for the rapid repression of TERT following the PT is that primary cultures of dermal fibroblasts taken from late embryonic skin (Figure 2B) have already completely repressed TERT expression. Taken together, these data indicate that the TERT gene is repressed very early in development. Thus, repression of TERT, the enzyme proposed by Olovnikov as essential for immortal replication, occurs shortly after PT resulting in the initiation of somatic restriction with a shift from immortal pluripotency to mortal somatic cells.

As discussed earlier, the limited replication capacity of somatic cell types due to the absence of telomerase expression ultimately leads to significant telomere erosion beginning in embryonic and fetal development, and continuing with further erosion during normal aging (and accelerated aging in the case of chronic injury) resulting in chronic tissue damage. Accordingly, this continued telomere erosion eventually results in critically short telomeres that trigger cell senescence, which is thought to limit the capacity of adult tissues to repair damage. In addition, senescent cells contribute to a loss of tissue integrity and increased inflammation via the expression catabolic enzymes such as metalloproteinases [31] and plasminogen activators [32], a phenomenon now designated as the senescence-associated secretory phenotype [33]. Therefore, cellular senescence as seen in the context of somatic restriction and antagonistic pleiotropy is a consequence of the loss of indefinite somatic cell replication via the loss of telomerase, which may provide an evolutionary advantage to the species by, for example, reducing tumor formation, but eventually leads to detrimental effects later in life such as the loss of tissue regeneration associated with aging.

\section{Inhibition of regeneration}

Assuming the hypotheses of Weismann and Williams are correct, the same selective pressures that inhibit replicative immortality could also restrict regeneration following the completion of embryogenesis. Therefore, the associated molecular pathways would potentially be important for understanding aging and possibly even cancer. Indeed, regeneration research has attracted the interest of researchers since the time of Thomas Hunt Morgan at the beginning of the 20th century [17]. These early models included the study of planaria and hydra as well as other invertebrate species, but these early studies were largely descriptive. Current research has extended the number of animal models to include urodeles, hemimetabolous insects, Xenopus and even mammals such as the African spiny mouse [34,35] and embryonic marsupials [36].

Perhaps the first advancement in our understanding of the molecular basis of the restriction of regenerative potential during development began with the search for genes that alter the developmental timeline (heterochrony 


\section{(A)}

(A) 9.0

TERT

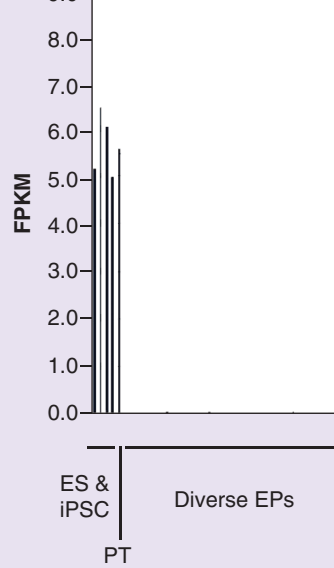

(B)

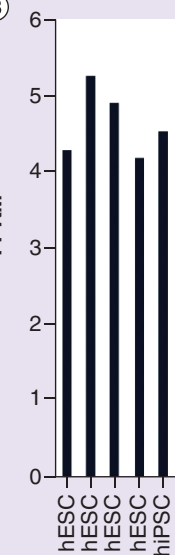

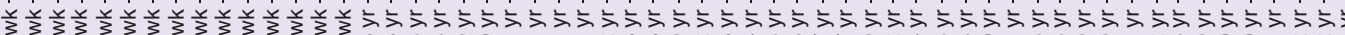

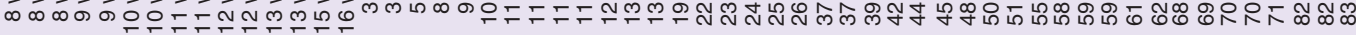

TERT
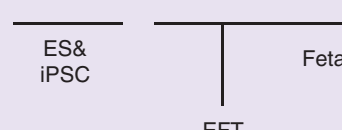

Fetal

EFT

Figure 2. TERT expression during differentiation in vitro and in vivo. The catalytic component of human telomerase (TERT) is an archetypal example of a gene with antagonistic pleiotropic effects in aging and cancer. (A) TERT is abundantly expressed in naturally immortal hES and iPSCs and in the majority of carcinomas, sarcomas and blood cell cancers. It is not expressed after the pluripotency transition in diverse hESC-derived embryonic progenitor cells, diverse human somatic cell types including cultured mesenchymal stem cells, vascular endothelial cells, neurons and glia, hepatocytes, osteochondral cell types as well as others; nor is TERT expressed in cultured epithelial cells such as keratinocytes, respiratory, mammary, prostate or other epithelia. (B) TERT is expressed in pluripotent stem cells, but not in human dermal fibroblasts from late-stage embryonic upper arm skin-derived fibroblasts cultured in vitro, or in skin from fetal or adult sources throughout the human lifespan.

AT: Adult transition; CA: Cancer cells; EP: Embryonic progenitors; hESC: Human embryonic stem cell; NT; Newborn transition; iPSC; Induced pluripotent stem cells; EFT: Embryonic-fetal transition; FPKM: Fragments per kilobase of transcript per million mapped reads; PT: Pluripotency transition.

genes) in Caenorhabditis elegans. One example is the lin41 (designated as TRIM71 in humans), a gene that is implicated in regulating cell proliferation [37], that is expressed early in the C. elegans development but repressed in the larval stages. Another example is the noncoding gene, let-7, whose transcribed product is a miRNA [38]. The let-7 miRNA appears to control temporal developmental events such as the transition from larval to adult states in the worm. It operates through the silencing of numerous target transcripts during differentiation and appears to be largely conserved in metazoans from $C$. elegans to Drosophila, mouse and man. Let-7 is thus thought to restrict regeneration during the process of development and, therefore, would need to be repressed in the early pre-EFT (embryonic-fetal transition) stages of development. 
Lin-28, which is expressed during embryogenesis and repressed following the EFT, is an RNA binding protein that regulates Let-7. Lin-28 appears to bind the primary and precursor forms of Let-7, thereby inhibiting its downstream processing. There are two homologs of Lin-28 present in the human species designated LIN28A and $L I N 28 B$. Both genes continue to be transcribed after TERT is repressed but they are eventually repressed at the EFT and, like TERT, they represent examples of genes that generate antagonistic pleiotropic effects in aging and cancer. Accordingly, LIN28B is abundantly expressed in naturally immortal hES cells and induced pluripotent stem cells (iPSC) but is generally repressed in a wide variety of hES cell-derived embryonic progenitor cell types except for embryonic vascular endothelial cells. LIN28B is not expressed in many different adult human somatic cell types including cultured mesenchymal stem cells, vascular endothelial cells, neurons and glia, hepatocytes and osteochondral cell types as well as various cultured epithelial cell types such as keratinocytes, respiratory, mammary, prostate or other epithelia (Figure 3A). In contrast, LIN28B is expressed at high levels in many carcinomas, sarcomas and blood cell cancers (Figure 3A) [39] but is expressed at much lower levels in normal cultured human skin fibroblasts than in cancer or pluripotent cells. These observations suggest a regenerative role for LIN28 that is restricted upon its repression around the time of the EFT (completion of 8 weeks of development) (Figure 3B).

A considerable number of reports substantiate a role for genes in the LIN28/Let-7 pathway not only as markers of the developmental stage of mammalian cells, but also as having a direct role in regulating regeneration and cancer. For example, the expression of LIN28A and/or LIN28B has been reported to increase axon regeneration in both peripheral and CNS neurons [40]; to increase regeneration of cartilage, skin and digits [41]; and to increase hematopoiesis [42]. Moreover, the LIN28/LET-7 target IGF2BP1 can even reprogram adult cells to express fetal hemoglobins [43]. In the urodeles, thyroid hormone appears to play a role in triggering metamorphosis. As such, Lin 28 has been reported to inhibit the expression of thyroid hormone target genes, delay development and prolong regenerative potential [44]. Consequently, given the importance of this pathway in developmental timing, it is interesting to ask whether activation of LIN28A and LIN28B or, alternatively, the abrogation of expression of the Let-7 family of miRNAs, can be used more broadly as a method of facilitating the induced tissue regeneration.

Metabolism also plays an essential role in development, regeneration and aging. Accordingly, developing embryonic tissues and presumably, regenerating tissues, have a greater need for anabolic precursors such as those originating from glycolysis as opposed to oxidative phosphorylation (OXPHOS). OXPHOS is necessarily minimized during embryogenesis by the rate-limiting lack of oxygen in the relatively anoxic environment of mammalian uterus. Interestingly, it has been recognized since the pioneering research of Otto Warburg, that a shift from glycolysis to OXPHOS occurs during early embryonic development and that a shift back to glycolysis, even in the presence of adequate oxygen, occurs in many cancers: a phenomenon known as the Warburg effect, or aerobic glycolysis [45]. These observations raise the question of what role the balance of glycolysis versus OXPHOS might play in the regenerative phenotype.

\section{The embryonic-fetal transition}

Placental mammals show a global loss of regenerative potential in numerous tissues during development in utero. Although the timing of the repression of scarless regeneration in humans depends on the tissue type, it is commonly associated with the EFT. In humans, this transition occurs at the completion of Carnegie Stage 23 (8 weeks of development). In the case of the mouse, this corresponds approximately with the close of Theiler Stage 23 (16 days post coitum $[\mathrm{dpc}])$. Consequently, regeneration during the EFT can be easily studied in marsupial mammals because the animals emerge and enter the pouch while still in the embryonic prefetal state where they are readily accessible for experimentation. Thus it has been determined that scarring begins around pouch day 9 near the EFT [36].

The in vitro model of human cell aging discovered by Hayflick, facilitated the discovery of the role of telomere dynamics in aging. Accordingly, the field of regeneration research is likely to increasingly benefit from the initial discovery of culture conditions for isolating and growing human pluripotent stem cells in vitro. Indeed, ES cells and iPSCs [28,46] provide an in vitro model system to study human cells in the germ-line state as well as embryonic somatic cells of diverse types that can be compared with their adult counterparts for analyzing in the molecular biology of the EFT under rigorously controlled conditions. Moreover, there are opportunities to study naive pluripotent cells, which are thought to be capable of robust germ-line transmission and therefore more closely aligned with the classic definition of ES cells. Additionally, primed ES cells or 'ES-like' cells also retain telomerase expression and generally maintain telomeres at germ-line length as well as pluripotency, but unlike naive cells commonly do not show the ability to be incorporated into the germ line such as when they are introduced into 


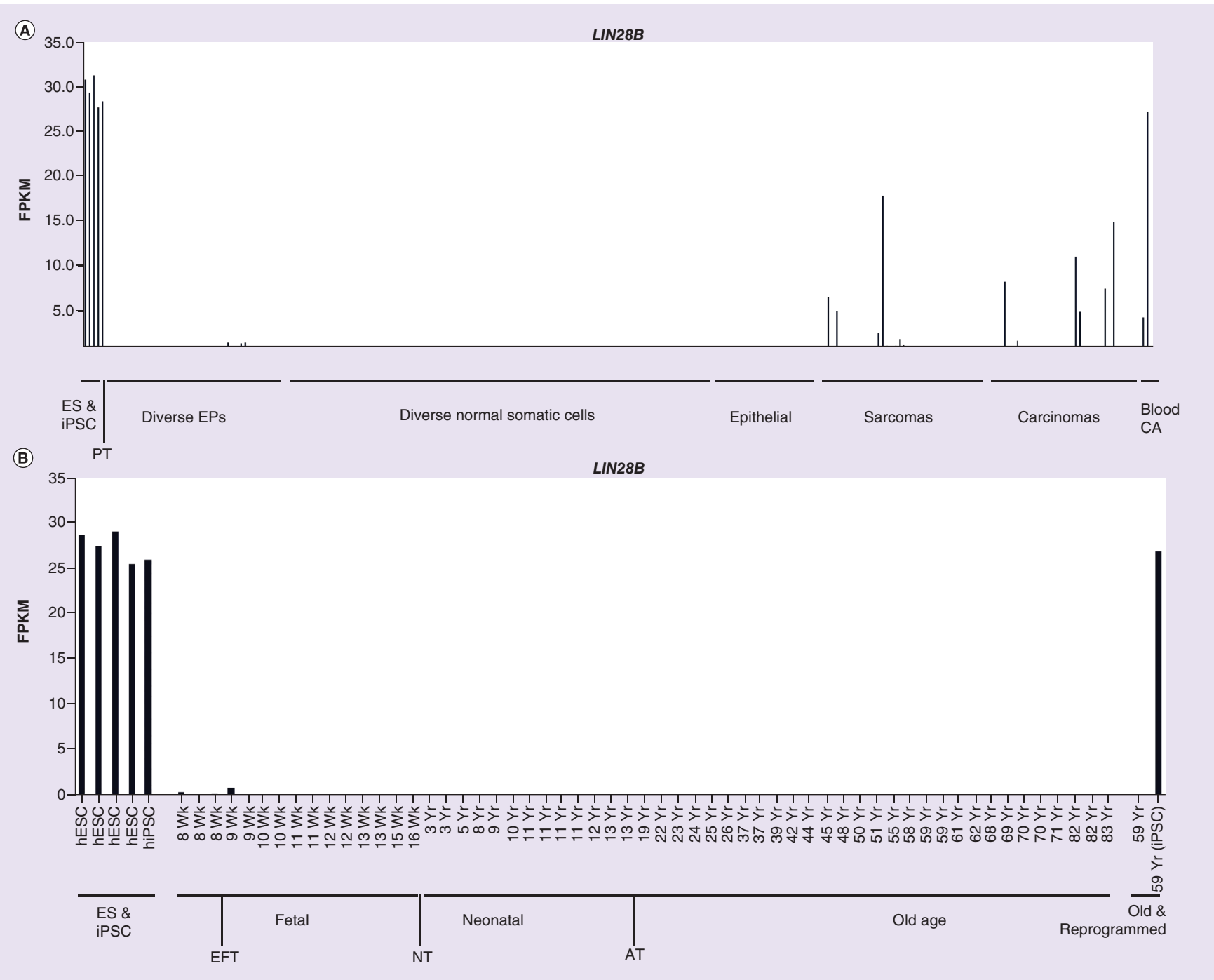

Figure 3. LIN28B expression during differentiation in vitro and in vivo. The gene encoding the RNA binding protein $L I N 28 B$ is an example of a gene repressed subsequent to TERT that also generates antagonistic pleiotropic effects in aging and cancer. (A) LIN28B is abundantly expressed in naturally immortal hES and iPSCs, but not expressed in most diverse hESC-derived embryonic progenitor cells except for embryonic vascular endothelial cells. LIN28B is neither expressed in diverse adult human somatic cell types including cultured mesenchymal stem cells, vascular endothelial cells, neurons and glia, hepatocytes, osteochondral cell types as well as others, nor is LIN28B expressed in cultured epithelial cells such as keratinocytes, respiratory, mammary, prostate or other epithelia; however, it is expressed at high levels in many carcinomas, sarcomas and blood cell cancers. (B) LIN28B is highly expressed in human pluripotent stem cells; it is expressed at lower levels in human dermal fibroblasts from late-stage embryonic upper arm skin-derived fibroblasts cultured in vitro, but not in skin from later fetal or adult sources throughout the human lifespan.

CA: Cancer cells; EP: Embryonic progenitors; hESC: Human embryonic stem cell; NT; Newborn transition; iPSC; Induced pluripotent stem cells; EFT: Embryonic-fetal transition; FPKM: Fragments per kilobase of transcript per million mapped reads; PT: Pluripotency transition.

chimeric blastocysts. Therefore, both naive and primed ES cells have not yet initiated the process of differentiation into the classic three germ layers (endoderm, mesoderm or ectoderm) and thus have not passed the PT making them valuable in vitro models for PT and EFT studies.

The ability to grow hES cells and iPSC in vitro facilitates the exploration of the transitions occurring during human development. However, their potential to differentiate into a very large complexity of diverse cell types can complicate a rigorous exploration of these processes. One approach to this problem is the clonal derivation of a large diverse library of clonal progenitor cell lines from hES or iPSC [30]. These pure and scalable clonal lines can be characterized and compared with their adult counterparts. Moreover, the use of artificial intelligence algorithms to compare hES, embryonic progenitor and adult normal and tumor cell line transcriptome data has 
facilitated the identification of robust markers of the PT and EFT stages [47]. One such marker, COX7A1, appears to begin expression in diverse stromal cell types at about the EFT. Accordingly, levels of expression of this gene are particularly high in adult skeletal and cardiac muscle, brown fat, and stromal cells, with a lower level in most epithelial cell types; however, there is no expression in many sarcomas and carcinomas [47]. Indeed, the repression of $C O X 7 A 1$ expression in numerous malignant cell types is consistent with the model of antagonistic pleiotropy. The role of COX7A1 in OXPHOS complex IV dimerization suggests that the onset of expression of the gene during development (post-EFT) plays a role in the repression of regeneration, whereas, the loss of expression in malignancy may play an important role in the Warburg effect and uncontrolled growth. Therefore, expression of COX7A1 following EFT might contribute in part to the lack of regenerative potential in most somatic tissues (hepatocytes, blood and epithelial cells being exceptions). Indeed, an important hallmark of aging is the progressive accumulation of interstitial fibrosis $[48,49]$ and the accumulation of fibrous connective tissue in many organs in old age may reflect this failed regenerative response to cumulative age-related injury. Further analysis of hES cells, iPSC cells and their derivatives in comparison with adult cells is likely to reveal additional molecular pathways involved in somatic restriction during developmental transitions such as PT and EFT.

\section{The neonatal transition}

The neonatal transition (NT) is designated here as the developmental transition resulting in profound global alterations in mammalian somatic gene expression that are associated with birth. This transition is marked by a dramatic decrease in growth rate. Indeed, body mass will increase dramatically by $>100$-fold during fetal development (EFT to NT) but growth will continue at a markedly slower rate following the NT. A survey of genes differentially expressed during the NT showed that many were associated with imprinted loci [50]. An example of this is the paternally imprinted fetal growth factor IGF2. Moreover, maternal and paternal imprints have been implicated in the regulation of not only placentation but fetal and perhaps postnatal growth as well [51]. Although endocrine factors such as thyroid hormone, IGF1 and IGF2, and growth hormone have been implicated in growth, the levels of these factors do not appreciably change with the cessation of growth in adulthood. Indeed, studies involving the transplantation of fetal rat tissues into adult rats suggest that factors such as growth hormone do not arrest the growth of the fetal rat tissue and indeed are necessary for differentiation [52]. These observations suggest that the regulation of growth at the NT results from an intrinsic developmental program as opposed to endocrine influences.

The mammalian heart appears to be under regulation by the NT and thus provides an important target organ model system to study the relationship between NT and regeneration. Unlike most organs, the heart retains an unusually high degree of regenerative potential after EFT, beyond NT and into the first postnatal week during which time cardiomyocytes begin to become binucleate [53,54]. Accordingly, ligation of the left anterior descending artery in 1 day old mice results in severe ischemic damage that is nevertheless completely regenerated scarlessly within 7 days. However, a similar injury induced in 7-day-old mice results in scarring instead of regeneration [55]. With regard to the mechanism, tracing studies argue in favor of the hypothesis that the regenerated myocardium in such perinatal mammals originates from the de-differentiation of mature myocardium as opposed to the competing theory that it originates from a mobilized pool of resident myocardial progenitors [56]. Therefore, in the case of the myocardium, the loss of regeneration occurring shortly after NT is likely not due to a loss or dysfunction of stem cells but rather a relative loss of plasticity in the differentiated cardiac cells (i.e., its ability to dedifferentiate and re-differentiate in response to injury). A better understanding of the NT and the mechanism of the regenerative capacity of the neonatal mammalian heart may enable new approaches to inducing regeneration in fully mature tissues and organs.

\section{The adult transition}

The adult transition (AT) occurs at the termination of growth in the reproductively mature adult and represents another transition where the somatic restriction hypothesis predicts molecular changes connected to aging and the loss of regeneration. Accordingly, numerous metazoans with developmental programs of continued growth following reproductive maturity (no AT) show negligible aging [5]. These observations led to 'Bidder's hypothesis' [1], which states that the growth arrest of animals upon reaching reproductive maturity is causally connected to aging (perhaps due to a change in metabolic rate) [57,58]. Accordingly, George Parker Bidder summarized his theory as, "we die, therefore, as an alternative to becoming giants" [59]. 
The molecular mechanisms underlying the AT have not been fully elucidated. However, differentially methylated regions in the genome have been implicated in the NT as well as in the later adult growth arrest (AT) in most mammalian species. In addition, the identification of the Hippo signaling pathway including genes such as MST4 and TEAD4 has provided new insights into the molecular biology of growth arrest in the AT. For example, both MST4 and TEAD4 have been reported to play a role in cell proliferation and cancer [60,61]. However, only a few studies have attempted to identify alterations in mediators of Hippo signaling that could play a role in aging [62]. Accordingly, a better understanding of the molecular mechanisms involved in the AT with respect to tissue growth arrest may also inform mechanisms that regulate regeneration with aging. It will also be important to understand the role of environment has in developmental transitions, regeneration and aging.

\section{Developmental plasticity in feast \& famine}

It seems likely that feast/famine cycles have had a profound effect on the evolution of metabolic regulation over evolutionary history. Consequently, many animals adapt to seasonal variations in caloric intake through their adipose tissue-based energy reserves. Additionally, they may catabolize somatic tissues such as muscle in severe conditions. Indeed, an extreme example is observed in certain animals such as planaria that dramatically regress in size in response to starvation [63]. However, when food becomes available again, their profound regenerative potential allows them to restore body mass. Remarkably, in the case of certain medusozoans, the adult can invert metamorphosis upon exposure to adverse conditions, wherein the pelagic medusa reverses the normal life cycle to become a juvenile benthic polyp [64]. Thus, in summary, numerous animal species have the potential to regress and regenerate to varying degrees in response to dietary restriction (DR). However, the molecular mechanisms controlling the induction of regenerative potential in DR are not fully understood.

Interestingly, DR is one of the most established means of modulating lifespan in a wide variety of species. Accordingly, one of the more reproducible observations about aging found in many laboratory animal models is that DR appears to forestall the onset of multiple markers of aging. Furthermore, DR decreases insulin/IGF-1 signaling (IIS), and increases activities of sirtuins and 5' AMPK, while decreasing mTOR activity, all of which are implicated in extending lifespan in model organisms $[65,66]$. The important role of metabolism in aging is consistent with the modulation of the activity of the nutrient-sensing kinases, AMPK and mTOR, by changes in the cell's nutritional state and the associated ATP levels even though cellular ATP concentration is generally well in excess of the Michaelis constant $\left(\mathrm{K}_{\mathrm{m}}\right)$ of most signaling pathway kinases. Therefore, it is intriguing to consider that DR may induce, to some extent, tissue regeneration. Indeed, this may be the pathway through which DR, or targeted modifications in proteins such as AMPK, mTOR or IIS molecules, can modulate lifespan.

\section{The somatic restriction theory of aging}

The diverse facets of aging often appear difficult to harmonize. For example, aging could be the result of telomerase repression in somatic cells and consequent telomere shortening and cell senescence. However, there are also environmental factors such as metabolic stress associated with IIS signaling and AMPK or mTOR signaling or lack of sirtuin histone deacetylase activity. We propose that the diverse observations related to aging can be largely harmonized through what we designate as the 'somatic restriction theory of aging'. We suggest that during development, global programmed alterations in gene expression occur in many cells and tissues of the body. Salient examples of the above include those spanning the PT, EFT, NT and AT, though it is likely others exist as well. These global alterations result in a progressive restriction of the differentiated state of somatic cells that benefits the organism in the pre- and perireproductive period by reducing the risks of developmental plasticity and its associated oncogenesis [67] as predicted by Williams.

In addition, we propose that the narrowing of facultative plasticity of the differentiated state of cells in somatic tissues leads to a progressive failure to regenerate tissues in response to damage with regeneration being replaced with interstitial fibrosis. We further postulate that the developmental loss of regenerative potential leads to a switch toward a program whereby DNA damage leads to senescence as opposed to apoptosis. In other words, if tissues can robustly regenerate, then the cells can undergo apoptosis when they experience significant DNA damage. However, if tissues have lost regenerative potential, then damaged cells are more likely to institute DNA checkpoint arrest and subsequent senescence instead of apoptosis as a means of maintaining tissue function. Last, with regard to molecular mechanisms, we suggest that somatic restriction is mediated epigenetically by an altered distribution of heterochromatin as proposed theoretically $[68,69]$ and empirically [70], and is therefore modifiable to some extent by feast/famine cycles, DR or potentially by partial reprogramming [71], and other novel interventions. 
Early events associated with somatic restriction, such as developmental shifts in gene expression patterns, are likely attributable to altered chromatin structure. Accordingly, mammalian gene expression, although regulated on several levels, is perhaps most significantly regulated by transcription factors binding proximal and distal regulatory elements such as promoters and enhancers located in topologically associating domains that are constrained by insulating elements such as a transcriptional repressor CCCTC-binding factor (CTCF) binding sites. An example of the role of topologically associating domains in regulating the expression of developmentally timed gene expression is seen in the hemoglobin locus, where changes in chromatin structure define the progressive expression of genes from embryonic $(\zeta$ and $\varepsilon$ ) to fetal $(\gamma)$ and adult $(\delta$ and $\beta$ ) globin genes [72]. Furthermore, an early determinative event in somatic restriction appears to be remodeling of the nuclear envelope and associated heterochromatin. Most eukaryotic cells are characterized by intranuclear euchromatin with heterochromatin being associated with the nuclear lamina on the inner surface of the nuclear membrane. Therefore, a shift in global gene expression may occur during embryonic development as the result of a shift in the composition of the protein components of the nuclear lamina from primarily lamin B receptor-dependent binding to a composite of lamin B receptor- and lamin A/C-dependent binding. Accordingly, LMNA, which is expressed at low levels in ES cells, is induced upon cell differentiation in vitro and is also induced on a protein level in a tissue-specific manner around the EFT in vivo [73]. These findings suggest possible role of chromatin structure and nuclear lamina structure in the somatic restriction of regenerative potential.

Epigenetic modifications are important mediators of chromatin binding to the nuclear envelope and subsequent alterations in gene expression patterns. Epigenetics was a term first introduced by Conrad Waddington in the mid-20th century. Robin Holliday defined epigenetics as 'the study of the mechanisms that impart temporal and spatial control on the activities of all those genes required for the development of a complex organism from the zygote to the adult' [74]. In addition, epigenetic modifications of chromatin and the resulting alterations in nuclear architecture in aging and cancer are of growing interest. Epigenetic modifications such as histone acetylation and methylation modulate chromatin binding and gene expression. Accordingly, it is now recognized that nuclear lamina-associated chromatin is silenced through lamina-associated histone-deacetylase activity [75]. Furthermore, the loss of acetylation and gain of methylation of histones such as trimethylation at lysines 9 and 27 of histone 3 (H3K9me3 and $\mathrm{H} 3 \mathrm{~K} 27 \mathrm{Me} 3)$ together with chromatin-binding proteins, such as HP1, are commonly associated with transcriptionally inactive heterochromatin [76]. In contrast, transcriptionally active euchromatin is commonly associated with the trimethylation at lysines 4 and 36 of histone 3 (H3K4me3 and H3K36me3) marks [76], or acetylation of histone 3 such as $\mathrm{H} 3 \mathrm{~K} 27 \mathrm{Ac}$.

Epigenetic modifications such as histone methylation and acetylation appear to play key roles in aging. Indeed, $\mathrm{H} 3 \mathrm{~K} 9 \mathrm{Me} 3$ and $\mathrm{H} 3 \mathrm{~K} 27 \mathrm{Me} 3$ marks are lost in numerous species with age supporting generalized loss of laminaassociated heterochromatin during aging [77,78]. Furthermore, regarding acetylation, silent information regulator SIR2, an nicotinamide adenine dinucleotide $(\mathrm{NAD})^{+}$-dependent histone deacetylase, extends budding lifespan in yeast and increases longevity in other species, in part by stabilizing DNA containing repetitive sequences [79]. These observations are consistent with a role of histone acetylation in aging, and the protective role of heterochromatin in maintaining the genomic integrity of repetitive sequences. Significantly, the segmental premature aging syndrome known as Hutchinson-Gilford Progeria Syndrome (HGPS) results from a dominant gain-of-function heterozygous mutation of codon 608 (G608G: GGC>GGT) in the lamin A/C (LMNA) gene, causing elevated expression of an isoform of lamin A, termed progerin. However, progerin may also play a role in normal aging as supported by the progerin-associated loss of nuclear $\mathrm{H} 3 \mathrm{~K} 9 \mathrm{Me} 3$ staining in fibroblasts during the course of normal aging both in vivo and in vitro [78]. Moreover, blocking progerin formation has been shown to restore normal H3K9Me3 levels in cultured fibroblasts [80], suggesting a potential upstream causal role for lamin A changes in the loss of heterochromatic $\mathrm{H} 3 \mathrm{~K} 9 \mathrm{Me} 3$. Thus, there is support for regulation of aging by a loss of epigenetic gene silencing that is mediated by lamin A and progerin.

Epigenetic regulation occurs at the beginning of early ES cell differentiation when lineage specificity is controlled at least in part by PRC2, containing the histone H3K27 methyltransferase EZH2. However, some 75-87\% of genes appear to be regulated by H3K27 methylation of associated CpG islands [81]. Therefore, it seems unlikely that this modification by itself is specific for regulating the subtle effects of transitions such as the EFT. Nevertheless, there is considerable evidence for a role of the methyltransferase in the somatic restriction of regeneration as well as aging. For example, EZH2 is implicated, together with Let-7 microRNAs in the NT of hematopoietic cells [82]. In addition, knock-down of utx-1, a histone demethylase specific for the EZH2 product, $\mathrm{H} 3 \mathrm{~K} 27 \mathrm{me}$, extends the lifespan of $C$. elegans [83]. Further, EZH2 is observed to decrease during human fibroblast senescence leading to 
increased expression of cyclin-dependent kinase inhibitors such as p16 (CDKN2A) [84]. Finally, we have observed that EZH2 is down-regulated in fibroblasts at the NT [WeST ET AL. 20I9, UnPublished Data].

The repressive histone modifications $\mathrm{H} 3 \mathrm{~K} 9 \mathrm{Me} 3$ and $\mathrm{H} 3 \mathrm{~K} 27 \mathrm{Me} 3$ also play an important role in the constitutive heterochromatin associated with repetitive DNA such as those sequences associated with telomeric repeats as well as subtelomeric DNA [85]. In addition to descriptive studies associating telomeres with heterochromatin, ectopically introduced telomeric repeats have been shown to generate relatively large regions $(\sim 225 \mathrm{~kb})$ of facultative heterochromatin marked by $\mathrm{H} 3 \mathrm{~K} 27 \mathrm{Me} 3$ [86]. It is important to note that telomeres are particularly vulnerable sites for DNA damage [87]. Indeed, they typically contain $2-15 \mathrm{~kb}$ of the double stranded tandemly-repeated sequence $(\text { TTAGGG) })_{n}$ with a terminal single-stranded overhang that is thought to circle back and integrate in a structure designated a t-loop [88]. Thus, telomeres contain a terminal single-stranded region that is sensitive to oxidative damage. However, they are shielded from some DNA repair enzymes by the shelterin complex [89], which normally would prevent them from being recognized as double-stranded breaks. Consequently, the relative loss of $\mathrm{H} 3 \mathrm{~K} 9 \mathrm{Me} 3$ and $\mathrm{H} 3 \mathrm{~K} 27 \mathrm{Me} 3$, and replacement of telomeric chromatin with acetylation marks with age may increase the likelihood of telomeric damage and/or the recognition of the single-stranded terminus as DNA damage leading to checkpoint signaling. Interestingly, decreased Sir2 expression in yeast results in increased acetylation of histones in the subtelomeric region and increased expression of the telomeric transcript called telomeric repeat-containing RNA (TERRA) that, in turn, can decrease telomere length [90]. Therefore, both histone acetylation and methylation may affect aging via their effects on telomeres. Indeed, the regulation of the heterochromatic state of telomeres may depend in part on $\mathrm{H} 3 \mathrm{~K} 9 \mathrm{Me} 3$ and $\mathrm{H} 3 \mathrm{~K} 27 \mathrm{Me} 3$. TERRA RNA has been reported to bind PRC2 facilitating the production of initially an $\mathrm{H} 3 \mathrm{~K} 27 \mathrm{Me} 3$ mark followed by H3K9Me3 marks and heterochromatin production [91]. These findings suggest that the PRC2 complex plays a role in telomere maintenance.

Other epigenetic changes in addition to the aforementioned age-related changes in histone modification and chromatin structure are widespread changes in methylated DNA at CpG sites. Changes in DNA methylation patterns with age have been reduced to a classifier capable of accurately predicting chronological age in blood and other cells [92]. These DNA methylation changes may be regulated by histone modifications such as H3K9Me3, which can alter the methylation of associated CpGs. Conversely, nonmethylated CpGs can affect histone modifications through zinc finger $\mathrm{CxxC}$ (ZF-CxxC) domain-containing proteins such as KDM2A and KDM2B [93]. Therefore, age-related histone modifications and DNA methylation may not be entirely independent phenomenon.

\section{Metabolism \& epigenetics}

As mentioned previously, mammalian homologs of yeast Sir2 such as the genes SIRT1 - SIRT7 act as NAD - $^{+}$ dependent 'erasers' that remove the acetyl group from histones. The dependency of SIRT1 on $\mathrm{NAD}^{+}$and its upregulation under DR conditions [94] have generated significant interest in its role in aging. However, while overexpressing Sirt1 in mice results in a healthier metabolic profile [95], only modest effects on lifespan have been reported to date [96]. In contrast to the 'erasers', histone 'writers' such as the lysine methyltransferases and lysine acetyltransferases also appear to be modulated to some extent by the metabolic state of cells. Accordingly, energy metabolites S-adenosyl-methionine and CoA provide the methyl groups and acetyl groups, respectively, for lysine methyltransferases and lysine acetyltransferases. Thus, the levels of both S-adenosyl-methionine and acetyl-CoA are examples of indicators of nutritional status that provide a mechanistic link between metabolism and the epigenetic modulation of gene expression [97,98]. Therefore, these links and others perhaps not yet identified may eventually explain the molecular basis of how fluctuations in caloric intake facilitate the adaptive response of regression and regeneration of body mass and the linkage, if any, of regeneration and lifespan extension in response to DR.

\section{Prospects for reprogramming senescence}

The demonstration of the feasibility of mammalian somatic cell de-differentiation by somatic cell nuclear transfer certainly shows that cell differentiation is entirely reprogrammable [99]. Moreover, while an initial analysis of telomere length in animals produced by somatic cell cloning suggested that telomere length was not restored in the animals [100], subsequent studies showed that somatic cell nuclear transfer could restore aged telomere lengths back to embryonic lengths or even longer [101]. Further, subsequent studies of telomere length dynamics in isogenic cells showed that induced pluripotency can also restore aged cell telomere restriction fragment (TRF) length back to that of cultured ES cells or even longer, though that is not always the case [102]. In addition, subsequent studies of the epigenetics of aging show that reprogramming by induced pluripotency can completely reset age-related DNA methylation changes [71]. 
The above findings logically lead to the question of whether reprogramming can transiently reverse the developmental age of cells only as far back as the Weismann barrier (pre-EFT) without inducing pluripotency and thus retaining the differentiated state of cells. Accordingly, it may be possible to use reprogramming approaches to unlock latent regenerative plasticity by transiently restoring cells to a 'pre-EFT' state. We have designated this strategy-iTR [47]. Given the importance of telomerase repression and telomere shortening with aging, we envision that iTR would also benefit from the transient induction of telomerase, therefore, whether or not telomere length is modulated in the course of iTR, we will refer to both strategies simply as iTR.

The challenge of iTR-related approaches is to reprogram the epigenetic age and other hallmarks of aging in adult cells in a manner that maintains cell identity while restoring the regenerative potential to pre-EFT levels but not increasing the risk of an embryo-onco phenotype and cancer. Accordingly, it is important to note that pluripotency-related pathways are increasingly recognized as playing an important role in oncogenesis [103]. Indeed, the expression of reprogramming factors such as Klf4, Oct4, Sox2 and Myc in mice has been reported to generate pluripotent cells and teratomas in vivo [104]. While teratomas are benign counterparts of malignant teratocarcinomas, teratomas themselves can adversely affect health. Therefore, Ocampo et al. used a strategy of limiting the expression of reprogramming factors (i.e., cyclic expression) to achieve a partially reprogrammed state, which resulted in prolonging lifespan in a mouse progeria model and in increased regeneration following muscle and pancreatic injury in naturally aged wild-type mice [105]. Moreover, they reported that numerous markers of aging were reversed including the restoration of young H3K9Me3 levels. Similarly, another study demonstrated that partial reprogramming in vivo resulted in improved wound healing with reduced scar formation [106]. Additionally, alternative approaches such as chemical induction of reprogramming may also influence regeneration potential. For example, a chemical cocktail shown to promote reprogramming in vitro had a protective effect against liver damage in vivo [107]. Although in their early stages, these studies and other ongoing efforts to reverse aging without triggering oncogenesis indicate the potential for the eventual translation of iTR approaches into simple, safe and cost-effective therapies. Indeed, iTR could have important implications for aging, epimorphic regeneration, and cancer diagnosis and therapy.

The developmental repression of regeneration affects multiple organ systems and iTR could benefit aged patients afflicted with degenerative conditions of numerous types. Cardiovascular disease appears to be a prime candidate for iTR because it is the greatest single cause of mortality in the USA and Europe, accounting for about 17.6 million deaths annually [108]. Indeed, the prevalence of heart failure in Americans in the years 2013-16 was approximately 6.2 million [108]. Cardiac regeneration is often seen in animals with relatively high capacity for regeneration such as zebrafish and axolotls or mice in the first neonatal week. As discussed earlier, detailed tracing studies suggest that cardiac regeneration occurs not through the mobilization of resident stem cells, but rather from regression of the differentiated cardiomyocyte into a precursor capable of proliferation and re-differentiation [56]. Therefore, is not unreasonable to assume that de-differentiation and re-differentiation, as opposed to the recruitment of resident stem cells, may be the mechanism of regeneration in many of these models of epimorphic regeneration. Further study is required to determine how pervasive this mechanism is in other organ systems.

\section{Discussion}

While certain evolutionarily primitive metazoans, and perhaps some vertebrates show no evidence of aging, mammals typically show an exponential increase in the risk of mortality with age. Mammalian aging can be viewed as a global developmental program in many cells and tissues in the body wherein somatic cells are progressively restricted in their capacity for immortal regeneration (Figure 4). Accordingly, these steps begin with the repression of the expression of the catalytic component of telomerase TERT resulting in the antagonistic pleiotropic effect of decreased risk of cancer early in life but a finite replicative lifespan of somatic cells leading to cell and tissue aging later in life. Other genes such as TRIM71 also appear to be repressed at or around the time of the PT, and the loss of expression may also play a role in restricting cell division. Moreover, subsequent developmental restrictions associated with the EFT, NT and AT may also play a role in the cadence of developmental changes that repress tissue regeneration following the completion of organogenesis and subsequent growth. In summary, widespread gene expression changes, like TERT repression, occur early in the life cycle, in many tissues within the soma and these early changes may have an antagonistic pleiotropic effect later in life leading to tissue disrepair associated with aging.

Conrad Waddington is credited with the model of cell differentiation and development pictured as a 3D landscape with increasingly restrictive canals representing the increasingly restrictive fate potential of somatic 


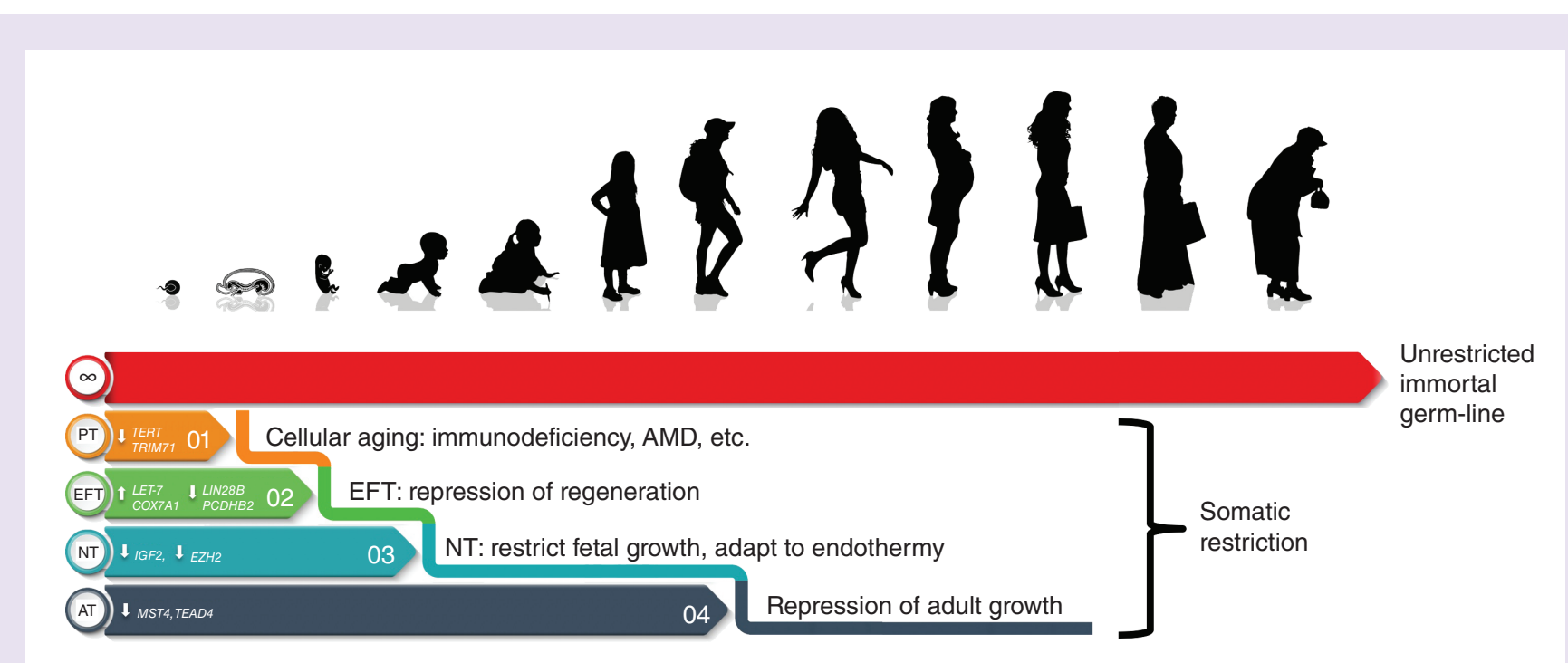

Figure 4. The stages of somatic restriction. While germ-line cells retain the potential to regenerate the species indefinitely, cells during the course of differentiation undergo progressive modifications that restrict either their capacity of indefinite replication (such as occurs at or around the time of the loss of pluripotency (pluripotency transition), or in subsequent steps that restrict their potential for regeneration and growth.

AMD: Age-related macular degeneration; AT: Adult transition; EFT: Embryonic-fetal transition; NT: Neonatal transition; PT: Pluripotency transition.

cells (Figure 5) [109]. The walls of the canals represent repressive mechanisms that constrain the plasticity of the differentiated state of the somatic cells wherein the topology and rigidity of cell fate is controlled by epigenetic influences tethered to gene expression (shown as cubes at the bottom of Figure 5). The staged developmental somatic restrictions (PT, EFT, NT and AT) are therefore seen as a narrowing and reinforcement of these walls to prevent cells from reversing their course or altering cell fate following, for example, the completion of embryonic development at the EFT. However, induced pluripotency can undo these restrictions, thus reversing the developmental age of cells to before the PT and thereby reactivating TERT expression, which restores the cells back to immortality. In contrast to induced pluripotency, iTR represents a novel strategy to revert somatic cells and tissues back only to the state of the cells before the EFT such that the plasticity of differentiated cells is restored as a means of promoting epimorphic regeneration.

The human body is thought to have considerable redundancy in homeostatic mechanisms. However, tissues within the body also have considerable interdependency. Accordingly, the time-dependent disrepair resulting in failure of just one tissue to maintain homeostasis can trigger cascades that throw other systems off balance. Indeed, this concept is supported by mathematical models of the resulting mortality curves that closely align with the empirical data. Furthermore, the models suggest that regenerative therapies that repair the initial degenerative triggering events will have a considerable impact on lifespan [110]. These theoretical predictions, combined with the power of technologies such as pluripotent stem cell-based cellular compositions, suggest that there may be a path not only to alleviate the high costs associated with the chronic degenerative diseases of aging [111] but also to extend human life expectancy. Since organisms with immortal somatic cells and full regenerative potential in the soma often show negligible senescence, the greatest advance may result from a strategy to implement transient induction of telomerase activity in combination with iTR.

\section{Conclusion}

Increasingly the theoretical framework underlying modern aging research is that progressive developmental transitions occur early in the life cycle that impact tissue regeneration and therefore aging in the soma. The theory of somatic restriction highlights the dichotomy of the immortal regenerative potential of the germ-line compared to the terminal mortal phenotype of most differentiated somatic cell types. The theory posits that somatic restriction occurs progressively in stages (pluripotency to differentiating embryo, embryo to fetal, fetal to neonate, and neonate 


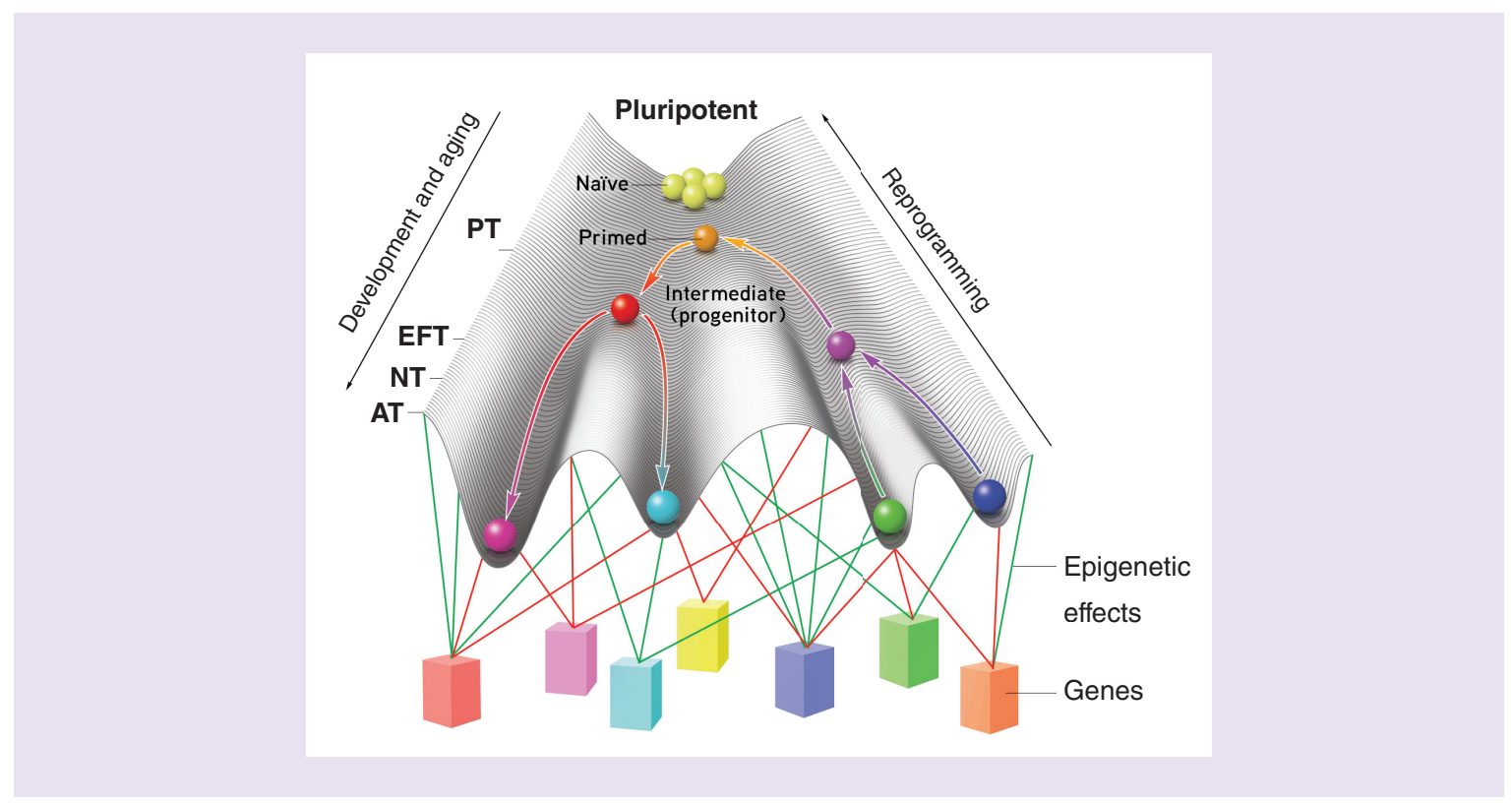

Figure 5. The Waddington epigenetic landscape of development, aging and reprogramming. Waddington proposed that genetic and epigenetic influences produced a developmental program of differentiation during development. This visual representation, based on Waddington's epigenetic landscape, illustrates somatic cell differentiation and somatic restriction at critical developmental transitions (PT, EFT, NT, AT) leading to loss of regenerative potential and aging. The transition from germ line-competent naive ES cells to primed ES cells leads to a reduced capacity for germ-line transmission but a retention of replicative immortality and the capacity to differentiate into all somatic cell types. The initiation of ES cell differentiation at the pluripotency transition leads to the production of diverse intermediate progenitors that generally lack telomerase expression. The subsequent differentiation of somatic cells and passage through the embryonic-fetal transition 'hardens' the pathways, reducing plasticity and thereby inhibiting regenerative potential. Induced pluripotent stem cell reprogramming is capable of restoring somatic cells back to either naive or primed immortality while iTR will likely be designed to partially reprogram cells back to a regenerative state while maintaining cell identity. The red line designates the Weismann barrier.

AT: Adult transition; EFT: Embryonic-fetal transition; ES: Embryonic stem; iTR: Induced tissue regeneration; NT: Neonatal transition; PT: Pluripotency transition.

Redrawn from [109].

to fully grown adult) and that many of these transitions occur globally in multiple organ systems. This conceptual framework provides a context for more detailed analytical studies of developmentally-regulated molecular pathways that were selected for reproductive fitness early in the life cycle, but result in homeostatic decline and failure of organ systems in aging adults (antagonistic pleiotropy). We conclude that modern molecular approaches to regenerative medicine such as reprogramming cells to pluripotency or partially reprogramming to induce tissue regeneration (iTR) effectively reverse most markers of aging and have significant potential for clinical application in aging.

\section{Future perspective}

The profound age-related demographic shift occurring in several of the largest industrialized countries of the world places an unprecedented strain on our healthcare systems. Fortunately, this tsunami of healthcare expenditures is contemporaneous with an equally unprecedented surge of advances in research into the biology of aging. This profound example of the confluence of an emerging supply and a rising demand presents the opportunity for innovative solutions to the healthcare crisis. Therefore, while many of the needs of the aged will be met on numerous fronts with diverse technologies, any therapeutic strategy aimed at the fundamental molecular biology of aging itself, that is also applicable to the diverse organ systems of the body, will almost certainly have the greatest impact on the healthcare industry and quality of life for the aging population. In vitro reprogramming is a promising technology, which can revert a donor cell of any age back to a state of pluripotency and immortality. Moreover, the fact that reprogramming also erases numerous markers of aging argues that cellular aging is reversable and thus opens the possibility of applying reprogramming as a therapeutic strategy to, for example, iTR in aging individuals. 
However, the in vivo translation of reprogramming technologies to clinical applications will require significant preclinical testing to insure safety, particularly with regard to the potential to increase cancer risk.

\section{Executive summary}

Somatic restriction \& antagonistic pleiotropy

- The advent of cell biology in the 19th century led to August Weismann's insightful hypothesis that heredity is transmitted by cells belonging to an immortal germ line, and that in most cases, the evolution of complex somatic cells and tissues is associated with a loss of immortal regeneration (somatic restriction) that results in aging. Thus, he correctly predicted the limited lifespan of cultured somatic cells due to cellular senescence.

- In 1957, George Williams hypothesized that aging evolved through a process of antagonistic pleiotropy, where traits benefitting fecundity early in life are selected for even though they simultaneously lead to age-related deterioration later in life.

- According to an emerging consensus view of the evolution of aging, primitive organisms showing negligible senescence have not traversed the Weismann barrier (loss of immortality and regeneration), while human somatic cell types cross the barrier early in development leading to downstream age-related change.

Somatic cell senescence

- The Weismann theory of somatic cell senescence was validated by Leonard Hayflick in the 1960s with the demonstration that normal diploid human somatic cell types display a finite replicative lifespan when cultured in vitro.

- Alexey Olovnikov first proposed that the Hayflick phenomenon was due to the absence of activity of a telomeric terminal transferase (telomerase) in somatic cells leading to progressive telomere shortening. In addition, he proposed that germ line and cancer cells can attain replicative immortality through the expression of an enzyme that can synthesize the telomeric DNA (the telomere hypothesis of cell aging and immortalization).

- The molecular cloning of the gene encoding the catalytic component of human telomerase (TERT) demonstrated that TERT is necessary and sufficient in most cell types to rescue cells from senescence.

Inhibition of regeneration

- Animals that retain replicative immortality and full regenerative potential as adults, such as hydra and planaria often show no evidence of aging.

- The search for genes that regulate developmental timing (heterochrony genes) in model organisms such as Caenorhabditis elegans led to the identification of miRNAs and RNA binding proteins such as let-7 and LIN28, which are highly conserved from worm to man.

- The LIN28/let-7 axis regulates diverse regenerative responses in tissues of all three germ layers; the decline of which is consistent with the theory of antagonistic pleiotropy. Notably, an embryonic pattern of gene expression in this axis appears to characterize numerous cancer cell types (the embryo-onco phenotype).

The embryonic-fetal transition

- Scarless regeneration appears to be markedly repressed in both placental and marsupial mammals at the embryonic-fetal transition, which occurs at about the ending of Carnegie Stage 23 (8 weeks of development) in human, and Theiler Stage $23(16 \mathrm{dpc})$ in mice.

- Clonal human pluripotent cell-derived progenitor cell lines and corresponding fetal and adult counterparts enable an in vitro model for the molecular analysis of human cell developmental transitions.

- Transcriptional analysis has defined markers of the embryonic-fetal transition including the downregulation of LIN28B and PCDHB2, and the upregulation of COX7A1.

The neonatal transition

- The neonatal transition (NT) is characterized by alterations in numerous imprinted genes.

- The rapid pace of growth in the embryonic and fetal stages is markedly curtailed after parturition.

- The heart is an example of an organ profoundly altered during the NT. Mammalian cardiac muscle can regenerate scarlessly following trauma for the first week after the NT but not thereafter.

The adult transition

- The adult transition is defined as the cessation of growth in the adult at reproductive maturity. Some animals including vertebrates that show little evidence of aging also display the capacity for unlimited growth.

- Bidder's hypothesis proposes that the cessation of growth upon reaching adulthood is causative in aging.

Developmental plasticity in feast \& famine

- Some animals such as planaria and jellyfish species can 'de-grow' in response to food scarcity.

- Humans have only a limited capacity to catabolize tissues in response to starvation and to regenerate upon the restoration of food supplies.

- Dietary restriction extends lifespan in numerous species through mechanisms that may include a limited induction of regenerative potential.

The somatic restriction theory of aging

- The somatic restriction theory proposes that aging is a global developmental program that progressively represses the capacity of most cells and tissues in the body for immortal regeneration. 
- The regulation of chromatin structure, such as the regions of DNA compacted in heterochromatin versus euchromatin, is controlled in part by association with the nuclear lamina.

- Certain mutations in the nuclear lamin gene, LMNA, can lead to premature aging of diverse tissue types.

- Epigenetic modifications such as the acetylation of lysines 9 and 27 of histone 3 can compromise the stability of regions of the genome with repetitive sequences such as those in the telomere and subtelomere.

Metabolism \& epigenetics

- The sirtuin family of histone deacetylases as well as the S-adenosyl-methionine-dependent histone methyltransferases and the acetyl-CoA-dependent histone acetyltransferases can translate metabolic states into epigenetic states that in turn alter gene expression states.

Prospects for reprogramming senescence

- The facile ability of somatic cell reprogramming to reset telomere length by reversing the repression of telomerase and its ability to reverse the epigenetic changes associated with aging suggest that it may be possible to induce tissue regeneration if the rejuvenation effects of reprogramming can be induced without reversion to pluripotency.

- Since pluripotent-specific gene expression is also observed in diverse types of cancer (the embryo-onco phenotype), in vivo reprogramming, if not tightly regulated, may increase the risk of teratomas or even malignancy.

- Induced tissue regeneration may have important applications in regenerative medicine, aging and cancer.

\section{Acknowledgments}

The authors thank I Turovets of Biotech Graphic Design for artistic support.

Financial \& competing interests disclosure

MD West, H Sternberg, J Janus, I Labat, NN Malik, ADNJ de Grey and D Larocca are employees of AgeX Therapeutics, Inc. (CA, USA). ADNJ de Grey is also an officer of SENS Research Foundation (CA, USA). KB Chapman is a student at Johns Hopkins University (MD, USA); NN Malik is also an employee of Juvenescence, Ltd (London, UK). The authors have no other relevant affiliations or financial involvement with any organization or entity with a financial interest in or financial conflict with the subject matter or materials discussed in the manuscript apart from those disclosed.

No writing assistance was utilized in the production of this manuscript.

\section{Open access}

This work is licensed under the Attribution-NonCommercial-NoDerivatives 4.0 Unported License. To view a copy of this license, visit http://creativecommons.org/licenses/by-nc-nd/4.0/

\section{References}

Papers of special note have been highlighted as: $\bullet$ of interest; $\bullet \bullet$ of considerable interest

1. Comfort A. The Biology of Senescence. Elsevier, NY, USA (1979).

2. De Grey AD. The desperate need for a biomedically useful definition of 'aging'. Rejuvenation Res. 16(2), 89-90 (2013).

3. Strehler BL.Time, Cells, and Aging. Demetriades Brothers, Larnaca, Cyprus (1999).

4. Kowald A. Lifespan does not measure ageing. Biogerontology 3(3), 187-190 (2002).

5. Finch CE. Longevity, Senescence, and the Genome. University of Chicago Press, IL, USA (1990).

6. Swahari V, Nakamura A. Speeding up the clock: the past, present and future of progeria. Dev. Growth Differ. 58(1), 116-130 (2016)

7. Pilling LC, Kuo CL, Sicinski K et al. Human longevity: 25 genetic loci associated in 389,166 UK biobank participants. Aging 9(12), 2504-2520 (2017).

8. Wang Y, Karlsson R, Lampa E et al. Epigenetic influences on aging: a longitudinal genome-wide methylation study in old Swedish twins. Epigenetics 13(9), 975-987 (2018).

9. Weismann A. Essays Upon Heredity and Kindred Biological Problems (2nd Edition). Clarendon Press, Oxford, UK (1891).

10. Martinez DE. Mortality patterns suggest lack of senescence in hydra. Exp. Gerontol. 33(3), 217-225 (1998).

11. Schaible R, Scheuerlein A, Danko MJ, Gampe J, Martinez DE, Vaupel JW. Constant mortality and fertility over age in Hydra. Proc. Natl Acad. Sci. USA 112(51), 15701-15706 (2015).

12. Fior J. Salamander regeneration as a model for developing novel regenerative and anticancer therapies. J. Cancer 5(8), 715-719 (2014).

13. Johnson CK, Voss SR. Salamander paedomorphosis: linking thyroid hormone to life history and life cycle evolution. Curr. Top. Dev. Biol. 103, 229-258 (2013). 
14. Flink IL. Cell cycle reentry of ventricular and atrial cardiomyocytes and cells within the epicardium following amputation of the ventricular apex in the axolotl, Amblystoma mexicanum: confocal microscopic immunofluorescent image analysis of bromodeoxyuridine-labeled nuclei. Anat. Embryol. (Berl.) 205(3), 235-244 (2002).

15. Cano-Martinez A, Vargas-Gonzalez A, Guarner-Lans V, Prado-Zayago E, Leon-Oleda M, Nieto-Lima B. Functional and structural regeneration in the axolotl heart (Ambystoma mexicanum) after partial ventricular amputation. Arch. Cardiol. Mex. 80(2), 79-86 (2010).

16. Levesque M, Villiard E, Roy S. Skin wound healing in axolotls: a scarless process. J. Exp. Zool. B Mol. Dev. Evol. 314(8), 684-697 (2010).

17. Morgan TH. Regeneration. Macmillan, NY, USA (1901).

18. Godwin JW, Rosenthal N. Scar-free wound healing and regeneration in amphibians: immunological influences on regenerative success. Differentiation 87(1-2), 66-75 (2014).

19. Mito T, Noji S. The two-spotted cricket Gryllus bimaculatus: an emerging model for developmental and regeneration studies. CSH Protoc. 3(12), 1-14 (2008).

20. Bryant DM, Sousounis K, Farkas JE et al. Repeated removal of developing limb buds permanently reduces appendage size in the highly-regenerative axolotl. Dev. Biol. 424(1), 1-9 (2017).

21. Alibardi L. Immunodetection of telomerase-like immunoreactivity in normal and regenerating tail of amphibians suggests it is related to their regenerative capacity. J. Exp. Zool. A Ecol. Genet. Physiol. 323(10), 757-766 (2015).

22. Hayflick L, Moorhead PH. The serial cultivation of human diploid cell strains. Exp. Cell Res. 25, 585-621 (1961).

23. Dell'orco RT, Mertens JG, Kruse PF Jr. Doubling potential, calendar time, and senescence of human diploid cells in culture. Exp. Cell Res. 77(1), 356-360 (1973).

24. Olovnikov AM. [Principle of marginotomy in template synthesis of polynucleotides]. Dok. Akad. Nauk SSSR 201(6), 1496-1499 (1971).

25. Bodnar AG, Ouellette M, Frolkis M et al. Extension of life-span by introduction of telomerase into normal human cells. Science 279(5349), 349-352 (1998).

- Validates the hypotheses that the telomeric clock limits cellular lifespan in somatic cells by showing forced expression of telomerase results in immortalization without oncogenic transformation.

26. Morales CP, Holt SE, Ouellette $\mathrm{M}$ et al. Absence of cancer-associated changes in human fibroblasts immortalized with telomerase. Nat. Genet. 21(1), 115-118 (1999).

27. Kim NW, Piatyszek MA, Prowse KR et al. Specific association of human telomerase activity with immortal cells and cancer. Science 266(5193), 2011-2015 (1994).

28. Thomson JA, Itskovitz-Eldor J, Shapiro SS et al. Embryonic stem cell lines derived from human blastocysts. Science 282(5391), 1145-1147 (1998).

29. Funk WD, Labat I, Sampathkumar J et al. Evaluating the genomic and sequence integrity of human ES cell lines; comparison to normal genomes. Stem Cell Res. 8(2), 154-164 (2012).

30. West MD, Sargent RG, Long J et al. The ACTCellerate initiative: large-scale combinatorial cloning of novel human embryonic stem cell derivatives. Regen. Med. 3(3), 287-308 (2008).

- Provides an in vitro model of early embryonic progenitor cell types using clonally pure and expandable cell lines derived from human pluripotent stem cells.

31. West MD, Pereira-Smith OM, Smith JR. Replicative senescence of human skin fibroblasts correlates with a loss of regulation and overexpression of collagenase activity. Exp. Cell Res. 184(1), 138-147 (1989).

32. West MD, Shay JW, Wright WE, Linskens MH. Altered expression of plasminogen activator and plasminogen activator inhibitor during cellular senescence. Exp. Gerontol. 31(1-2), 175-193 (1996).

33. Coppe JP, Patil CK, Rodier F et al. Senescence-associated secretory phenotypes reveal cell-nonautonomous functions of oncogenic RAS and the p53 tumor suppressor. PLoS Biol. 6(12), 2853-2868 (2008).

34. Mehta AS, Singh A. Insights into regeneration tool box: an animal model approach. Dev. Biol. doi:10.1016/j.ydbio.2019.04.006 (2019) (Epub ahead of print).

35. Seifert AW, Kiama SG, Seifert MG, Goheen JR, Palmer TM, Maden M. Skin shedding and tissue regeneration in African spiny mice (Acomys). Nature 489(7417), 561-565 (2012).

36. Armstrong JR, Ferguson MW. Ontogeny of the skin and the transition from scar-free to scarring phenotype during wound healing in the pouch young of a marsupial, Monodelphis domestica. Dev. Biol. 169(1), 242-260 (1995).

37. Chang HM, Martinez NJ, Thornton JE, Hagan JP, Nguyen KD, Gregory RI. Trim71 cooperates with microRNAs to repress Cdkn1a expression and promote embryonic stem cell proliferation. Nat. Commun. 3, 923 (2012).

38. Reinhart BJ, Slack FJ, Basson M et al. The 21-nucleotide let-7 RNA regulates developmental timing in Caenorhabditis elegans. Nature 403(6772), 901-906 (2000).

39. Balzeau J, Menezes MR, Cao S, Hagan JP. The LIN28/let-7 pathway in cancer. Front. Gen. 8, 31 (2017). 
40. Wang XW, Li Q, Liu CM et al. Lin28 signaling supports mammalian PNS and CNS axon regeneration. Cell Rep. 24(10), 2540-2552 (2018).

41. Shyh-Chang N, Zhu H, Yvanka De Soysa T et al. Lin28 enhances tissue repair by reprogramming cellular metabolism. Cell 155(4), 778-792 (2013).

-• Expression of an embryonic-fetal transition marker that is normally expressed in pluripotent cells and during embryonic development results in improved tissue regeneration.

42. Yuan J, Nguyen CK, Liu X, Kanellopoulou C, Muljo SA. Lin28b reprograms adult bone marrow hematopoietic progenitors to mediate fetal-like lymphopoiesis. Science 335(6073), 1195-1200 (2012).

43. De Vasconcellos JF, Tumburu L, Byrnes C et al. IGF2BP1 overexpression causes fetal-like hemoglobin expression patterns in cultured human adult erythroblasts. Proc. Natl Acad. Sci. USA 114(28), E5664-E5672 (2017).

44. Faunes F, Gundermann DG, Munoz R, Bruno R, Larrain J. The heterochronic gene Lin28 regulates amphibian metamorphosis through disturbance of thyroid hormone function. Dev. Biol. 425(2), 142-151 (2017).

45. Warburg O. On the origin of cancer cells. Science 123(3191), 309-314 (1956).

46. Takahashi K, Tanabe $\mathrm{K}$, Ohnuki M et al. Induction of pluripotent stem cells from adult human fibroblasts by defined factors. Cell 131(5), 861-872 (2007).

-• Drawing on previous work with somatic cell nuclear transfer, demonstrates for the first time that somatic cell differentiation can also be reversed in vitro using as little as four ectopically expressed transcription factors to reprogram the cells to an embryonic pluripotent state.

47. West MD, Labat I, Sternberg $\mathrm{H}$ et al. Use of deep neural network ensembles to identify embryonic-fetal transition markers: repression of COX7A1 in embryonic and cancer cells. Oncotarget 9(8), 7796-7811 (2018).

48. Kramann R, Humphreys BD. Kidney pericytes: roles in regeneration and fibrosis. Sem. Nephrol. 34(4), 374-383 (2014).

49. Kapetanaki MG, Mora AL, Rojas M. Influence of age on wound healing and fibrosis. J. Pathol. 229(2), 310-322 (2013).

50. Lui JC, Finkielstain GP, Barnes KM, Baron J. An imprinted gene network that controls mammalian somatic growth is down-regulated during postnatal growth deceleration in multiple organs. Am. J. Physiol. Regul. Integr. Comp. Physiol. 295(1), R189-R196 (2008).

51. Lui JC, Forcinito P, Chang M, Chen W, Barnes KM, Baron J. Coordinated postnatal down-regulation of multiple growth-promoting genes: evidence for a genetic program limiting organ growth. FASEB J. 24(8), 3083-3092 (2010).

52. Cooke PS, Yonemura CU, Russell SM, Nicoll CS. Growth and differentiation of fetal rat intestine transplants: dependence on insulin and growth hormone. Biol. Neonate 49(4), 211-218 (1986).

53. Soonpaa MH, Field LJ. Survey of studies examining mammalian cardiomyocyte DNA synthesis. Circ. Res. 83(1), 15-26 (1998).

54. Porrello ER, Mahmoud AI, Simpson E et al. Transient regenerative potential of the neonatal mouse heart. Science 331(6020), 1078-1080 (2011).

55. Haubner BJ, Adamowicz-Brice M, Khadayate $S$ et al. Complete cardiac regeneration in a mouse model of myocardial infarction. Aging 4(12), 966-977 (2012).

56. Zhang Y, Mignone J, Maclellan WR. Cardiac regeneration and stem cells. Physiol. Rev. 95(4), 1189-1204 (2015).

57. Minot CS. The Problem of Age, Growth, and Death; a Study of Cytomorphosis, Based on the Lectures at the Lowell Institute. G.P. Putnam's Sons, NY, USA (1908).

58. Rubner M. Das Problem det Lebensdaur und seiner beziehunger zum Wachstum und Ernarnhung. Oldenberg, Munich, Germany (1908).

59. Bidder GP. Growth and death. Proc. Linn. Soc. LDN 137(1), 17-20 (1925).

60. Thompson BJ, Sahai E. MST kinases in development and disease. J. Cell Biol. 210(6), 871-882 (2015).

61. Chen L, Chan SW, Zhang X et al. Structural basis of YAP recognition by TEAD4 in the hippo pathway. Genes Dev. 24(3), 290-300 (2010).

62. Watt KI, Harvey KF, Gregorevic P. Regulation of tissue growth by the mammalian hippo signaling pathway. Front. Physiol. 8, 942 (2017).

63. Felix DA, Gutierrez-Gutierrez O, Espada L, Thems A, Gonzalez-Estevez C. It is not all about regeneration: planarians striking power to stand starvation. Semin. Cell Dev. Biol. 87, 169-181 (2019).

64. Piraino S, Boero F, Aeschbach B, Schmid V. Reversing the life cycle: medusae transforming into polyps and cell transdifferentiation in Turritopsis nutricula (Cnidaria, Hydrozoa). Biol. Bull. 190(3), 302-312 (1996).

65. Kimura KD, Tissenbaum HA, Liu Y, Ruvkun G. daf-2, an insulin receptor-like gene that regulates longevity and diapause in Caenorhabditis elegans. Science 277(5328), 942-946 (1997).

66. Templeman NM, Murphy CT. Regulation of reproduction and longevity by nutrient-sensing pathways. J. Cell Biol. 217(1), 93-106 (2018).

67. Suva ML, Riggi N, Bernstein BE. Epigenetic reprogramming in cancer. Science 339(6127), 1567-1570 (2013).

68. Villeponteau B. The heterochromatin loss model of aging. Exp. Gerontol. 32(4-5), 383-394 (1997). 
69. Imai S, Kitano H. Heterochromatin islands and their dynamic reorganization: a hypothesis for three distinctive features of cellular aging. Exp. Gerontol. 33(6), 555-570 (1998).

70. Janssen A, Colmenares SU, Karpen GH. Heterochromatin: guardian of the genome. Annu. Rev. Cell Dev. Biol. 34, 265-288 (2018).

71. Olova N, Simpson DJ, Marioni RE, Chandra T. Partial reprogramming induces a steady decline in epigenetic age before loss of somatic identity. Aging Cell 18(1), e12877 (2019).

-• Shows epigenetic age is completely reversed in early stages of reprogramming before pluripotency is established.

72. Huang P, Keller CA, Giardine B et al. Comparative analysis of three-dimensional chromosomal architecture identifies a novel fetal hemoglobin regulatory element. Genes Dev. 31(16), 1704-1713 (2017).

73. Jia Y, Vong JS, Asafova A et al. Lamin B1 loss promotes lung cancer development and metastasis by epigenetic derepression of RET. J. Exp. Med. doi:10.1084/jem.20181394 (2019) (Epub ahead of print).

74. Holliday R. DNA methylation and epigenetic inheritance. Philos. Trans. R Soc. Lond. B Biol. Sci. 326(1235), 329-338 (1990).

75. Finlan LE, Sproul D, Thomson I et al. Recruitment to the nuclear periphery can alter expression of genes in human cells. PLoS Genet. 4(3), e1000039 (2008).

76. Shilatifard A. Chromatin modifications by methylation and ubiquitination: implications in the regulation of gene expression. Ann. Rev. Biochem. 75, 243-269 (2006)

77. Larson K, Yan SJ, Tsurumi A et al. Heterochromatin formation promotes longevity and represses ribosomal RNA synthesis. PLoS Genet. 8(1), e1002473 (2012).

78. Scaffidi P, Misteli T. Lamin A-dependent nuclear defects in human aging. Science 312(5776), 1059-1063 (2006).

79. Kennedy BK, Gotta M, Sinclair DA et al. Redistribution of silencing proteins from telomeres to the nucleolus is associated with extension of life span in S. cerevisiae. Cell 89(3), 381-391 (1997).

80. Scaffidi P, Misteli T. Reversal of the cellular phenotype in the premature aging disease Hutchinson-Gilford progeria syndrome. Nat. Med. 11(4), 440-445 (2005).

81. Comet I, Riising EM, Leblanc B, Helin K. Maintaining cell identity: PRC2-mediated regulation of transcription and cancer. Nature Rev. Cancer 16(12), 803-810 (2016)

82. Oshima M, Hasegawa N, Mochizuki-Kashio M et al. Ezh2 regulates the Lin28/let-7 pathway to restrict activation of fetal gene signature in adult hematopoietic stem cells. Exp. Hematol. 44(4), 282-296 (2016).

83. Maures TJ, Greer EL, Hauswirth AG, Brunet A. The H3K27 demethylase UTX-1 regulates C. elegans lifespan in a germline-independent, insulin-dependent manner. Aging Cell 10(6), 980-990 (2011).

84. Ito T, Teo YV, Evans SA, Neretti N, Sedivy JM. Regulation of cellular senescence by polycomb chromatin modifiers through distinct DNA damage- and histone methylation-dependent pathways. Cell Rep. 22(13), 3480-3492 (2018).

85. Jezek M, Green EM. Histone modifications and the maintenance of telomere integrity. Cells 8(2), 199-211 (2019).

86. Jamieson K, McNaught KJ, Ormsby T, Leggett NA, Honda S, Selker EU. Telomere repeats induce domains of H3K27 methylation in Neurospora. Elife 7, e31216 (2018).

87. Ahmed W, Lingner J. Impact of oxidative stress on telomere biology. Differentiation 99, 21-27 (2018).

88. Griffith JD, Comeau L, Rosenfield S et al. Mammalian telomeres end in a large duplex loop. Cell 97(4), 503-514 (1999).

89. De Lange T. Shelterin: the protein complex that shapes and safeguards human telomeres. Genes Dev. 19(18), 2100-2110 (2005).

90. Maicher A, Kastner L, Dees M, Luke B. Deregulated telomere transcription causes replication-dependent telomere shortening and promotes cellular senescence. Nucleic Acids Res. 40(14), 6649-6659 (2012).

91. Montero JJ, Lopez-Silanes I, Megias D, FF M, Castells-Garcia A, Blasco MA. TERRA recruitment of polycomb to telomeres is essential for histone trymethylation marks at telomeric heterochromatin. Nat. Commun. 9(1), 1548 (2018).

92. Horvath S. DNA methylation age of human tissues and cell types. Genome Biol. 14(10), R115 (2013).

- Establishes a method for estimating biological age based on changes of DNA methylation patterns with increasing age.

93. Blackledge NP, Thomson JP, Skene PJ. CpG island chromatin is shaped by recruitment of ZF-CxxC proteins. Cold Spring Harb. Perspect. Biol. 5(11), a018648 (2013).

94. Nemoto S, Fergusson MM, Finkel T. Nutrient availability regulates SIRT1 through a forkhead-dependent pathway. Science 306(5704), 2105-2108 (2004).

95. Bordone L, Cohen D, Robinson A et al. SIRT1 transgenic mice show phenotypes resembling calorie restriction. Aging Cell 6(6), 759-767 (2007).

96. Satoh A, Brace CS, Rensing $\mathrm{N}$ et al. Sirt 1 extends life span and delays aging in mice through the regulation of Nk2 homeobox 1 in the DMH and LH. Cell Metab. 18(3), 416-430 (2013).

97. Sivanand S, Viney I, Wellen KE. Spatiotemporal control of acetyl-CoA metabolism in chromatin regulation. Trends Biochem. Sci. 43(1), 61-74 (2018). 
98. Gao T, Diaz-Hirashi Z, Verdeguer F. Metabolic signaling into chromatin modifications in the regulation of gene expression. J. Mol. Sci. 19(12), 4108-4122 (2018).

99. Wilmut I, Schnieke AE, McWhir J, Kind AJ, Campbell KH. Viable offspring derived from fetal and adult mammalian cells. Nature 385(6619), 810-813 (1997).

100. Shiels PG, Kind AJ, Campbell KH et al. Analysis of telomere lengths in cloned sheep. Nature 399(6734), 316-317 (1999).

101. Lanza RP, Cibelli JB, Blackwell C et al. Extension of cell life-span and telomere length in animals cloned from senescent somatic cells. Science 288(5466), 665-669 (2000).

102. Vaziri H, Chapman KB, Guigova A et al. Spontaneous reversal of the developmental aging of normal human cells following transcriptional reprogramming. Regen. Med. 5(3), 345-363 (2010).

- It was initially unclear whether telomere length was restored to embryonic length in reprogrammed cells. Demonstrates that reprogramming human cells to pluripotency can reset telomere length to embryonic levels using an isogenic system.

103. Hepburn AC, Steele RE, Veeratterapillay R et al. The induction of core pluripotency master regulators in cancers defines poor clinical outcomes and treatment resistance. Oncogene 38(22), 4412-4424 (2019).

104. Abad M, Mosteiro L, Pantoja C et al. Reprogramming in vivo produces teratomas and iPS cells with totipotency features. Nature 502(7471), 340-345 (2013).

105. Ocampo A, Reddy P, Martinez-Redondo P et al. In vivo amelioration of age-associated hallmarks by partial reprogramming. Cell 167(7), 1719-1733 e1712 (2016).

-• First demonstration that in vivo reprogramming can extend lifespan in progeria mouse model and restore regeneration in aged mice.

106. Doeser MC, Scholer HR, Wu G. Reduction of fibrosis and scar formation by partial reprogramming in vivo. Stem Cells 36(8), 1216-1225 (2018).

107. Tang Y, Cheng L. Cocktail of chemical compounds robustly promoting cell reprogramming protects liver against acute injury. Protein Cell 8(4), 273-283 (2017).

108. Benjamin EJ, Muntner P, Alonso A et al. Heart disease and stroke statistics-2019 update: a report from the American Heart Association. Circulation 139(10), e56-e528 (2019).

109. Waddington CH. The Strategy of the Genes: A Discussion of Some Aspects of Theoretical Biology. George Allen \& Unwin, London, UK (1957).

110. Phoenix C, De Grey AD. A model of aging as accumulated damage matches observed mortality patterns and predicts the life-extending effects of prospective interventions. Age (Dordr.) 29(4), 133-189 (2007).

111. West MD, Binette F, Larocca D, Chapman KB, Irving C, Sternberg H. The germline/soma dichotomy: implications for aging and degenerative disease. Regen. Med. 11(3), 331-334 (2016). 